\title{
Measurement of Higgs to WW in the all-jet final state at CEPC $\sqrt{s}=250 \mathrm{GeV}$
}

\section{Mila Pandurovića,}

${ }^{a}$ Vinca Institute of Nuclear Sciences, University of Belgrade, National institute of the Republic of Serbia

Mike Petrović a Alasa 12-14, Beograd, Srbija

E-mail: milap@vinca.rs

The most important pillar in the physics case of future electron-positron colliders in high energy physics is the measurement of the Higgs boson, with its main goal to precisely measure its properties and to probe potential of associated new physics. All next generation electron positron facilities in high energy physics will make use of the Higgsstrahlung Higgs production channel. The physics potential of CEPC for measurement of the cross-section times branching ratio of subdominant decay $H \rightarrow W W^{*}$ is presented. The Higgsstrahnlung Higgs production channel is used, at the center of mass energy of $250 \mathrm{GeV}$. The fully hadronic decay, containing six soft-jets in the final state is of great importance for the detector design, jet pairing and reconstruction as showing the capability of CEPC for $\mathrm{W} / \mathrm{Z}$ separation. The analysis is performed in full simulation.

40th International Conference on High Energy physics - ICHEP2020

July 28 - August 6, 2020

Prague, Czech Republic (virtual meeting)

${ }^{*}$ Speaker 


\section{Introduction}

The discovery of the Higgs boson in 2012, has completed the Standard model of the particle physics. Since then, various measurements at the Large hadron collider LHC showed good agreement with the Standard model predictions. A possible path to the Beyond Standard model (BSM) physics, could be the precision measurements of the Higgs couplings to the Standard model (SM) particles, where the subtle difference in these couplings are foreseen by different BSM models. The necessary precision for the exploration of these deviations would require the percent or sub percent coupling precision levels [2]. As specified in the European strategy update for 2020, the High luminosity LHC's (HL-LHC) precision for these type of BSM searches could be enhanced by the additional collider facilities, Higgs boson factories, as the next step in the particle physics after the LHC era.

CEPC (Circular electron positron collider) [3] is one of four proposed international electron positron colliders, which is intended to be build in China. The collider is to be operated with the nominal center-of-mass energy of $\sqrt{s}=240 \mathrm{GeV}$ and the foreseen integrated luminosity of $5.6 \mathrm{ab}^{-1}$. This will amount to about one million of the Higgs boson events during the seven years running time. The CEPC is also foreseen to operate at the $\mathrm{Z}$ pole $(\sqrt{s}=91 \mathrm{GeV})$ and near the WW threshold $(\sqrt{s}=160 \mathrm{GeV})$ with the corresponding respective running times of 1 and 2 years, and $10^{7}$ and $10^{11}$ accumulated $\mathrm{Z}$ and $\mathrm{W}$ events. Also, the CEPC could be upgraded to a proton-proton collider with a center-of-mass energy around $100 \mathrm{TeV}$.

\section{All jet final state of the Higgs decay through the $\mathrm{W}$ boson pair}

At the nominal center of mass energy of CEPC, the dominant Higgs production channel is the Higgsstrahlung (HZ), which allows for the determination of the absolute Higgs to the $\mathrm{Z}$ boson coupling [4]. Also using the absolute Higgs to Z boson coupling as well as the Higgs boson width, which can be measured with high precision at CEPC [3], the subsequent determination of all the absolute couplings can be performed.

We are investigating the potential of CEPC for the measurement of the cross section times branching fraction of the Higgs boson decay to the pair of the $\mathrm{W}$ bosons in Higgsstrahlung. Of particular interest to access this coupling is utilizing all jets final state, since this channel tests the detector capability to resolve the electroweak bosons in multijet environment. The high branching fraction of the Higgs boson decay to hadronic final state, through the pair of $\mathrm{W}$ bosons, as well as high integrated luminosity delivers the abundant signal sample of more than $10^{4}$ signal events.

\section{Event simulation and reconstruction}

Signal and background are generated using Whizard 1.95 [5]. The samples are fully simulated with Mokka [6], at the $\sqrt{s}=250 \mathrm{GeV}$ and $m_{H}=125 \mathrm{GeV}$. The radiation effects, including initial and final state radiation are considered in the generation. The events are reconstructed with the particle flow based reconstruction software, Arbor [7].

The SM backgrounds consists of purely hadronic final states of two-fermion processes, 
$e e^{-} \rightarrow q \bar{q}$, and four-fermion processes of type $e e^{-} \rightarrow q \bar{q} q \bar{q}$. Semileptonic and leptonic events are not included in the background samples since it has been shown that survival probability of these types of backgrounds using multivariate methods for signal and background separation for this type of Higgs decay final states, is at the permille level or better [8].

Signal signature is characterized with six jets in the final state coming from the hadronic decays of both $\mathrm{W}$ bosons from the Higgs decay as well as the $\mathrm{Z}$ boson. The signal events possess high centrality, with jets which are widely spread, thus potentially overlapping. Therefore the most sensitive parts of the event selection are jet clustering and jet pairing. For jet clustering the $k_{t}$ clustering algorithm is used, which minimizes the ambiguities related to particle clustering within overlapping cones in multijet events [9]. Jets are clustered with high jet opening $(\mathrm{R}=1.5)$.

After clustering the reconstruction of bosons in the event is performed. The reconstruction is based on the minimization of the $\chi^{2}$ which takes into account the masses of the real bosons in the event - the real $\mathrm{W}$ boson, the $\mathrm{Z}$ and the Higgs boson. Six reconstructed jets in the event are paired into three di-jets. The di-jet masses are compared to the corresponding reference boson masses, with the additional constraint on the invariant mass of a quadri-jet in the event, which should correspond to reference mass of the Higgs boson:

$$
\chi^{2}=\frac{m_{i j}-m_{W}}{\sigma_{W}^{2}}+\frac{m_{k l}-m_{Z}}{\sigma_{Z}^{2}}+\frac{m_{i j m n}-m_{H}}{\sigma_{H}^{2}},
$$

where $m_{i j}$ refer to di-jet masses, and $m_{Z}, m_{W}$ and $m_{H}$ are the reference masses of the $\mathrm{Z}$, the $\mathrm{W}$ boson and the Higgs boson [1] and the $\sigma_{V}, \mathrm{~V}=\mathrm{W}, \mathrm{Z}, \mathrm{H}$, are the corresponding convolution of the boson width and the detector resolution. The detector resolution is set to $4 \%$ of the boson mass [10]. Figure 1 shows the reconstructed invariant masses of real $\mathrm{W}$ boson, the $\mathrm{Z}$ and the Higgs boson, where the clear peak distinction between the $\mathrm{W}$ and the $\mathrm{Z}$ boson masses is shown. Thus this particular final state shows the capability of the CEPC for separation of $\mathrm{W}$ and the $\mathrm{Z}$ boson in the highest jet multiplicity environment at the Higgs boson factories.

The reconstruction of the invariant masses of the corresponding bosons is influenced by multiple sources: the relative vicinity of the $\mathrm{W}$ and the $\mathrm{Z}$ boson mass and corresponding boson widths resulting in the intrinsic overlap, while the detector resolution, jet clustering and jet pairing are inducing the additional overlap in the reconstructed boson masses. The quantification of the separate factor's influence is studied on the four jet final states and are given in [10].

The discrimination of signal against the background is performed in two steps, where the first step, the preselection, uses the static cut separation. The primary aim of the preselection is the reduction of the high cross section background, in the first place, two fermion final states. The correlation between the energy of boson and the angle between the jets that comprise the boson is used to construct two preselection variables, 'energyTheta" (Figure 2), one for the $\mathrm{Z}$ and one for the $\mathrm{W}$ boson. The preselection uses the minimal set of variables: the number of particles in the final state and 'energyTheta', for the $\mathrm{Z}$ and the $\mathrm{W}$ boson. Preselection variables have high background reduction efficiency and moderate 


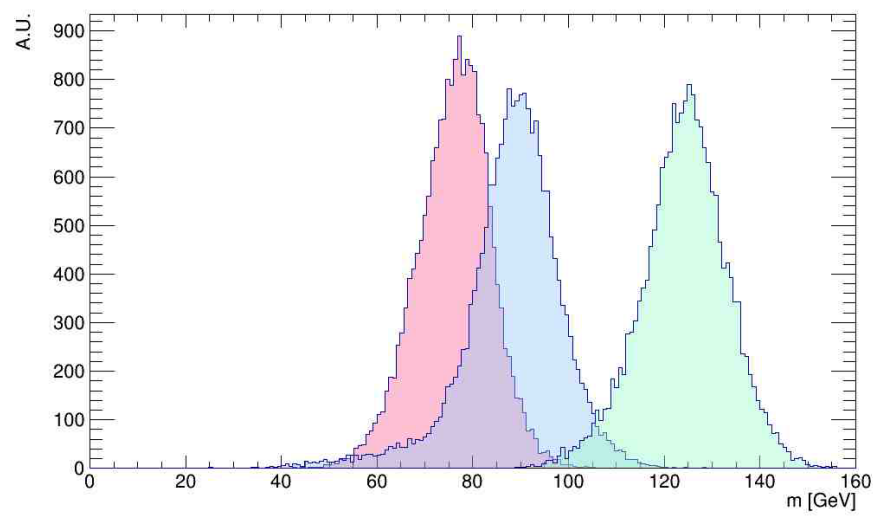

Figure 1: Distributions of the reconstructed dijet invariant masses, of the $\mathrm{Z}$ boson (pink), the $\mathrm{W}$ boson (blue) and the Higgs boson (green) candidates, for six jet signal final state, $H Z \rightarrow H \rightarrow$ $W W^{*} \rightarrow q \bar{q} q \bar{q}, Z \rightarrow q \bar{q}$.
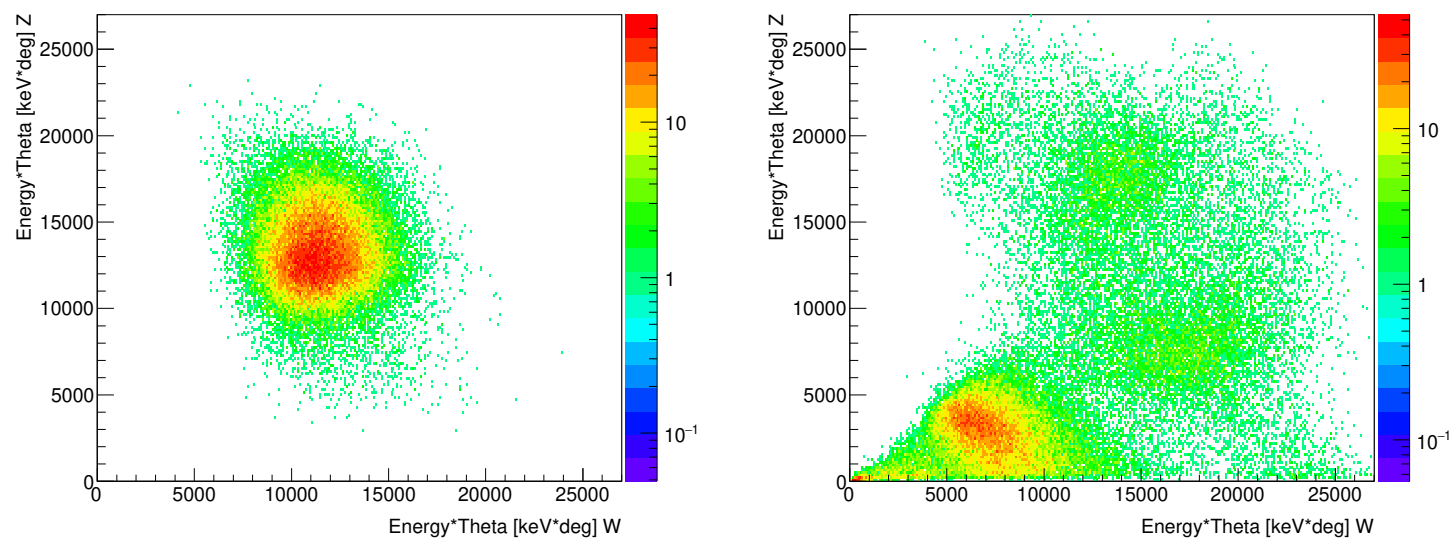

Figure 2: The preselection 'energyTheta' variable given for signal (left) and two-fermion background (right). The distribution of 'energyTheta' for $\mathrm{W}$ boson versus 'energyTheta' for the $\mathrm{Z}$ boson.

mutual correlation. The static cuts were optimized with respect to the signal efficiency, with the minimal signal efficiency of $70 \%$.

The final step in signal and background separation is the multivariate analysis (Boosted decision tree). Five groups of sensitive variables which exploit the different event features are used: the event shape variables, the jets transitions, the flavor tagging variables, kinematic variables and invariant masses of real bosons in the event. The correlations among the variables belonging to different groups are low. The most efficient variables are the event thrust and jet transitions for high jet multiplicities, around the number of jets in the signal $\left(y_{56}, y_{67}, y_{45}\right)$.

Due to the nature of the hadronic final states, with the mentioned sources of ambiguities 
Table 1: Expected relative precision on the $\sigma(\mathrm{ZH}) \times \mathrm{BR}\left(\mathrm{H} \rightarrow W W^{*}\right)$ measurement from CEPC dataset of $5.6 \mathrm{ab}^{-1}$.

\begin{tabular}{llr}
\hline ZH final state & & Relative statistical uncertainty [\%] \\
\hline \hline$Z \rightarrow e e^{-}$ & $H \rightarrow W W^{*} \rightarrow l v l^{\prime} v, l v q \bar{q}$ & 2.6 \\
$Z \rightarrow \mu \mu^{-}$ & $H \rightarrow W W^{*} \rightarrow l v l^{\prime} v, l v q \bar{q}$ & 2.4 \\
$Z \rightarrow \nu \nu$ & $H \rightarrow W W^{*} \rightarrow l v q \bar{q}, q \bar{q} q \bar{q}$ & 1.5 \\
$Z \rightarrow q \bar{q}$ & $H \rightarrow W W^{*} \rightarrow q \bar{q} q \bar{q}$ & 1.7 \\
\hline & Combined & 0.9 \\
\hline \hline
\end{tabular}

in the signal reconstruction, this type of final state lacks the clear signal tagging variable, in comparison to the leptonic or invisible $\mathrm{Z}$ decays of Higgsstrahlung Higgs production channel. This results in low signal efficiency after the final selection of 29\%. However, this is compensated with high integral luminosity foreseen at the CEPC, and results in the relative statistical uncertainty of the measurement of $1.9 \%$ for $5.0 \mathrm{ab}^{-1}\left(1.7 \%\right.$ for $\left.5.6 \mathrm{ab}^{-1}\right)$.

This result has entered the global Higgs fit. The list of the considered final states, as well as the obtained relative statistical uncertainties are given in the table, classified according to the $\mathrm{Z}$ decay Table 1 .

As can be seen from the table, combined fit of certain semileptonic and leptonic final states of $H \rightarrow W W^{*}$ decay [11], led to the improved precision of the measurement $H \rightarrow W W^{*}$ to $0.9 \%$. The result could be further improved by analyses of the complete set of final states of this particular decay.

\section{Conclusion}

This analysis is part of global effort to determine the potential of the CEPC for the precision measurement of the Higgs boson couplings to the Standard model particles. In this talk, the measurement accuracy, $\Delta(\sigma \cdot B R) /(\sigma \cdot B R)$, of the Higgs decay to a $W W$ decay, using the Higgsstrahlung Higgs production channel is presented. Fully hadronic final states is considered. This particular final state shows the capability of the CEPC for separation of $\mathrm{W}$ and the $\mathrm{Z}$ boson in the highest jet multiplicity environment at the Higgs boson factories. The study is performed with nominal center of mass energy $\sqrt{s}=250 \mathrm{GeV}$ and integrated luminosity of $5 \mathrm{ab}^{-1}$. The analysis is based on a full detector simulation using Geant4. The obtained result for the relative statistical uncertainty of the $\sigma(H Z) \cdot B R\left(H \rightarrow W W^{*}\right)$ is $1.9 \%\left(1.7 \%\right.$ for $\left.5.6 \mathrm{ab}^{-1}\right)$. The result has entered the global Higgs fit, which improved the measurement of the $H \rightarrow W W^{*}$ decays, to $0.9 \%$.

\section{Acknowledgments}

The author acknowledges the support received from the Ministry of Education, Science and Technological Development (Republic of Serbia). The author would also like to thank 
the CEPC software working group for providing the simulation and reconstruction tools and producing the Monte Carlo samples used in this study and the CEPC Physics and Detectors working group, Dr M. Ruan and Dr J.Guimaraes da Costa of IHEP (The Institute of High Energy Physics (IHEP) of the Chinese Academy of Sciences) for the invitation to perform the analysis.

\section{References}

[1] M. Tanabashi et al., Particle Data Group, Phys. Rev. D 98, 030001, (2018).

[2] M.Peskin, Searching for New Physics using Precision Standard Model Measurements, arXiv:2003.05435, (2020).

[3] The CEPC Study Group, CEPC Conceptual Design Report, Volume 1 - Accelerator, arXiv:1809.00285, Volume 2 - physics and detector, arXiv:1811.10545, (2018).

[4] Z. Chen, M. Ruan, Higgs recoil analysis and Higgs width measurement at CEPC, Proceedings of the 38th International Conference on High Energy Physics (ICHEP 2016), PoS ICHEP2016, 432 (2016).

[5] W. Kilian, T. Ohl and J. Reuter, WHIZARD: Simulating Multi-Particle Processes at LHC and ILC, Eur. Phys. J. C 71 1742, DOI:10.1140/epjc/s10052-011-1742-y, (2011).

[6] P. Mora de Freitas and H. Videau, Detector simulation with MOKKA/GEANT4: Present and future, The International Workshop on Linear Colliders (LCWS 2002).

[7] M. Ruan, Arbor, a new approach of the Particle Flow Algorithm, arXiv:1403.4784, (2014).

[8] M. Pandurovic, Physics Potential for the Measurement of $\sigma(\mathrm{HZ}) \times \mathrm{BR}\left(\mathrm{H} \rightarrow W W^{*}\right)$ at the $250 \mathrm{GeV}$ ILC, Talk presented at the International Workshop on Future Linear Colliders (LCWS2018), Arlington, Texas, 22-26 October 2018, C18-10-22, (2019).

[9] S. Catani, Y.L. Dokshitzer, M.H. Seymour, and B.R. Webber, Longitudinally invariant Kt clustering algorithms for hadron hadron collisions, Nucl.Phys. B406:187224, (1993).

[10] Y. Zhu, M. Ruan, Performance study of the full hadronic WW and ZZ events separation at the CEPC, Eur. Phys. J. C79 274,(2019).

[11] Fenfen An et al, Precision Higgs physics at the CEPC, Chinese Phys. C 43043002 (2019). 